\title{
essais dynamiques de fondations en centrifugeuse
}

\author{
PAUL LEGUAY \\ Ingénieur \\ Centre d'Etudes Scientifiques et Techniques d'Aquitaine*
}

PIERRE MORLIER

Professeur

Laboratoire de Génie Civil de l'Université de Bordeaux I, I.U.T. A** E.R.A. 769 du C.N.R.S.

\section{JOËL RIVIERE}

Chercheur

Laboratoire de Génie Civil de l'Université de Bordeaux I, I.U.T. A** E.R.A. 769 du C.N.R.S.

\section{INTRODUCTION}

La conception parasismique des constructions exige, pour l'introduire dans les codes de calcul, la connaissance quantitative de l'interaction dynamique solstructure; si cette connaissance est largement acquise pour un sol élastique homogène [2] [3], il n'en est pas de même si l'on veut tenir compte de l'hétérogénéité induite dans un sol réel par la pesanteur.

Le but de cette étude est d'apporter une réponse à ce genre de question; pour simuler la pesanteur il nous fut possible d'utiliser la centrifugeuse très performante du Centre d'Etudes Scientifiques d'Aquitaine dans le cadre d'un travail de thèse [6].

Nous décrivons donc d'abord cette centrifugeuse puis les essais particuliers de vibration de fondation; nous

(*) 33830 - Le Barp.

(**) 33405 - Talence Cedex. rapporterons ensuite l'approche théorique du problème et enfin nous montrerons comment on peut établir une notion de module équivalent et comment celui-ci est défini pour un sol semi-infini pulvérulent soumis à la pesanteur.

On ne s'intéresse ici qu'à la composante verticale du mouvement de vibration des fondations dans le but de simplifier l'exposé.

\section{ESSAIS DE VIBRATION DES FONDA- TIONS EN CENTRIFUGEUSE}

Du fait de la complexité des lois de comportement des sols, il apparaît nécessaire [1] [4] de réaliser des modèles réduits de fondations qui conservent le matériau (échelle des masses spécifiques égale à l'unité) et les contraintes, c'est-à-dire tels que, les contraintes en des points homologues du modèle et de la structure étant égales, les lois rhéologiques en ces points soient les mêmes. 
En soumettant, en centrifugeuse, une maquette à une accélération égale à $\mathrm{g}^{*}$ fois l'accélération de la pesanteur, les conditions ci-dessus imposent, d'après les lois de la dynamique (1), que:

- l'échelle des longueurs et des déplacements soit égale à $\mathrm{g}^{*-1}$,

- l'échelle des temps à $\mathrm{g}^{*-1}$,

- l'échelle des fréquences à $\mathrm{g}^{*}$,

- l'échelle des vitesses à 1 .

- l'échelle des masses à $\mathrm{g}^{*-3}$.

Ainsi, pour un $\mathrm{g}^{*}$ maximum de 100 sur la centrifugeuse du C.E.S.T.A., un accélérogramme de la maquette doit avoir une durée 100 fois plus faible et une amplitude 100 fois plus grande que la durée et l'amplitude d'un accélérogramme réel.

\subsection{La centrifugeuse Latecoere du C.E.S.T.A.}

Depuis quelques années, la centrifugeuse du C.E.S.T.A est utilisée pour la mécanique des sols, en particulier pour l'étude de la réponse à des séismes de lensemble sol-structure [5] [6].

D'après l'étude réalisée par Pilot [4], cette centrifugeuse est largement compétitive, par ses performances accélération de $100 \mathrm{~g}$ sur des modèles de 2 tonnes -, au plan mondial; on peut en particulier y réaliser des tests combinés d'accélération statique (avec conditionnement éventuel de vide ou de température) et d'essais vibratoires ou de chocs (séismes) [6].

La centrifugeuse comporte un bras dissymétrique d'une masse de 40 tonnes, portant (fig. 1)
- à une extrémité les quatre points d'amarrage des bâtis supportant les spécimens, dont une nacelle pivotante utilisée pour les essais de mécanique des sols,

- à l'autre extrémité, le contre-poids.

Le bras repose sur un ensemble «moyeu-support " lié par un réducteur à quatre moteurs à courant continu de $350 \mathrm{CV}$, chacun.

Les principales caractéristiques de cette centrifugeuse sont:

- la distance du pivot au centre de gravité des spécimens: 10 mètres;

- l'accélération maximale pour une masse de 2 tonnes: $1000 \mathrm{~ms}^{-2}$;

- la vitesse de rotation pour l'accélération maximale: $96 \mathrm{tr} / \mathrm{min}$;

- le temps de montée minimale pour atteindre $1000 \mathrm{~ms}^{-2}: 60 \mathrm{~s}$;

- la précision de la régulation de la vitesse angulaire: $10^{-2}$ rad. $\mathrm{s}^{-1}$, soit $2 \mathrm{~ms}^{-2}$ pour $1000 \mathrm{~ms}^{-2}$.

Une centrale d'acquisition multivoie placée au pivot, permet le traitement de 200 voies d'extensométrie statique; 10 voles d'extensométrie dynamique (bande passante $10 \mathrm{KH}_{2}$ ) et 24 voies accélérométriques (acquisition des phénomènes transitoires) complètent les moyens de mesure.

\subsection{Les essais de vibration de fondations en centrifugeuse}

Le sol de la maquette est contenu dans une cellule en aluminium, de dimension $1,3 \times 0,8 \times 0,4 \mathrm{~m}$, dont les parois internes sont recouvertes d'isomode afin que les

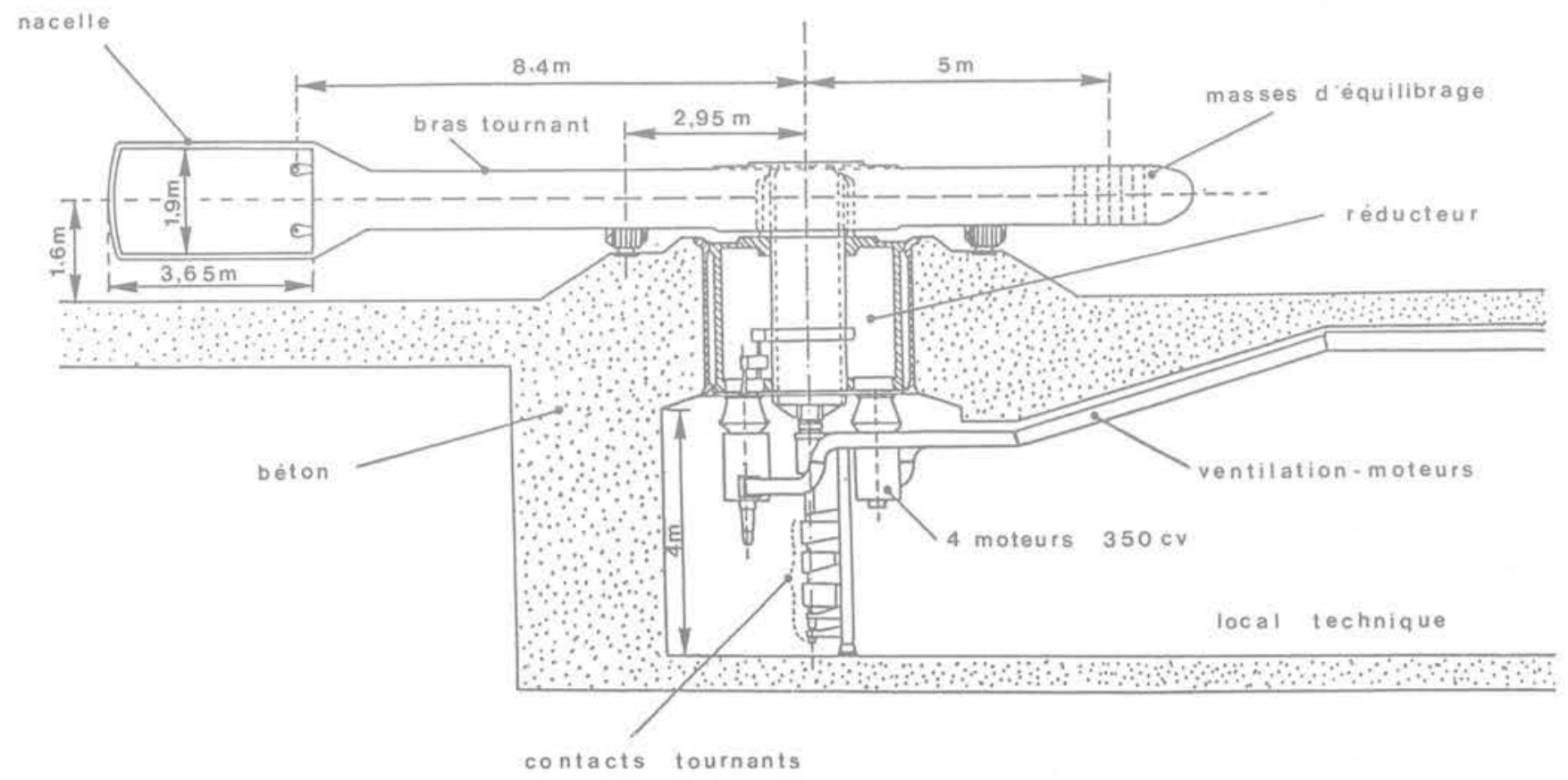

Fig. 1. - Centrifugeuse du C.E.S.T.A. 
vibrations parasites transmises par les parois soient absorbées.

Le sol est un sable fin de Fontainebleau, à granulométrie serrée, saturé d'eau: le remplissage en sable de la cellule est effectué en saupoudrant ce sable d'une hauteur de deux mètres afin d'obtenir un poids spécifique élevé (densité sèche: 1,6 ); la saturation en eau se fait en écoulement ascendant très lent.

Les modèles de fondation sont trois plaques circulaires d'aluminium de dimensions différentes:

$\begin{array}{llc} & \text { diamètre } & \text { poids } \\ \mathrm{P}_{1} & 0,183 \mathrm{~m} & 10,20 \mathrm{~N} \\ \mathrm{P}_{2} & 0,200 \mathrm{~m} & 16,60 \mathrm{~N} \\ \mathrm{P}_{3} & 0,253 \mathrm{~m} & 26,10 \mathrm{~N}\end{array}$

(dans les poids sont inclus ceux de l'excitateur et des capteurs).

L'excitateur est un moteur à balourd qui peut tourner au maximum à $400 \mathrm{~Hz}$; le poids du balourd est de $15 \mathrm{mN}$.

La force vibratoire est mesurée par un capteur inséré entre le socle du moteur et la plaque circulaire; elle est de la forme $Q=Q_{0} e^{i \omega t}$, avec, bien sûr,

$\mathrm{Q}_{0}(\mathrm{~N})=1,27510^{-6} \omega^{2}$

où $\omega$ est la pulsation.

L'accélération verticale du modèle a été mesurée à l'aide de deux accéléromètres diamétralement opposés sur la plaque dont on fait la demi-somme des indications.

\section{THÉORIE DE LA VIBRATION DES FONDATIONS SUR SOL ÉLASTIQUE}

Le sol est supposé être représenté par un milieu semi-infini, à surface libre horizontale, homogène, isotrope et élastique, caractérisé par son module de cisaillement $G$, son coefficient de Poisson $v$ et sa masse spécifique $\rho$. La fondation est représentée par une masse oscillant verticalement qui produit à la surface du sol une contrainte verticale uniformément répartie sur une surface circulaire de rayon $r_{0}$.

Avec ces hypothèses [2,3], le déplacement vertical z au centre de la fondation est exprimée par:

$z=P_{0} \frac{e^{i \omega t}}{G_{0}}\left(f_{1 v}+i f_{2 v}\right)$

où $\mathrm{P}_{0}$ est l'amplitude de la force totale verticale appliquée sur la fondation, et $f_{1 v}$ et $f_{2 \gamma}$ sont les fonctions de déplacement de Reissner (pour ce type de sollicitation).

Ces fonctions de déplacement peuvent être exprimées en fonction du coefficient de Poisson du sol et d'un nombre sans dimension, proportionnel à la fréquence,

$$
a_{0}=\omega r_{0} \sqrt{\rho / G}=\omega r_{0} / V_{s}
$$

où $V_{s}$ est la célérité des ondes de cisaillement dans le sol. La figure 2 donne les valeurs de ces fonctions

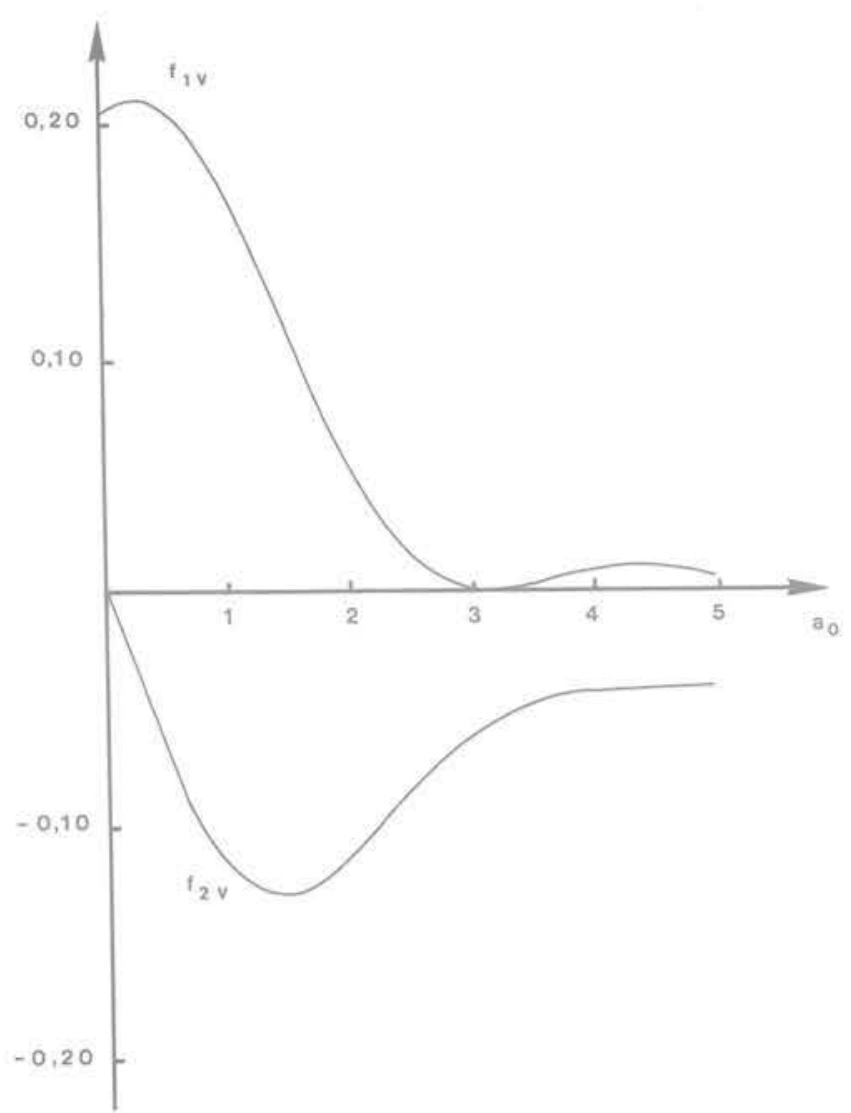

Fig. 2a. - Fonctions de déplacement de Reissner pour $v=0,25[6]$.

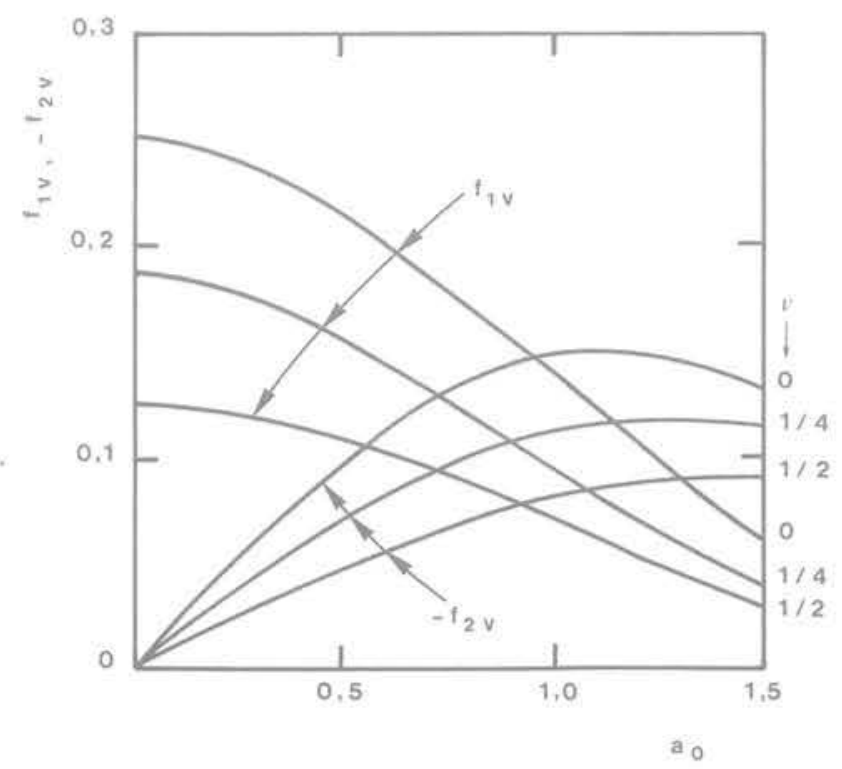

Fig. 2b. - Fonctions de déplacement pour une semelle circulaire rigide [3]. 
pour le coefficient de Poisson de 0,25 qui a été retenu dans cette étude ainsi que diverses valeurs de $v$ mais avec une plage plus réduite de $\mathrm{a}_{0}$.

A partir de l'expression (1) du déplacement vertical, Hsieh va donner l'équation du mouvement sous une forme plus traditionnelle; considérons (fig. 3) dans un
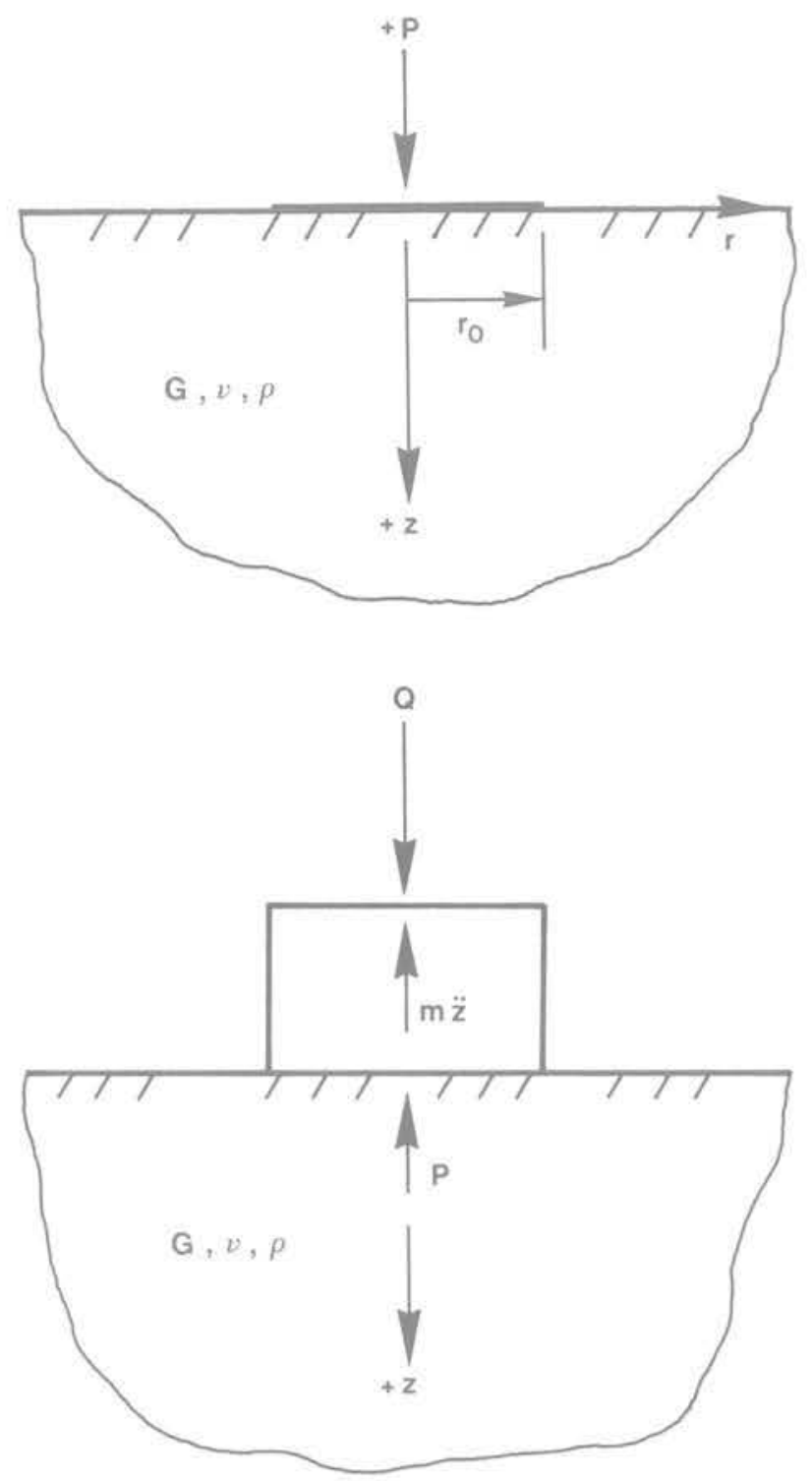

Fig. 3. - Notation pour les équations de Hsieh.

premier temps un disque sans masse de rayon $r_{0}$ à la surface du semi-infini et soumis à une charge verticale périodique $\mathrm{P}=\mathrm{P}_{0} e^{i \omega t}$; en dérivant l'équation (1) par rapport au temps, il vient

$\frac{d z}{d t}=\frac{P_{0} \omega e^{i \omega t}}{G r_{0}}\left(i_{1 v}-f_{2 v}\right)$

en combinant les relations (1) et (3) de façon à éliminer les termes imaginaires, on obtient: $f_{1 v} \omega z-f_{2 v} \frac{d z}{d t}=\frac{P \omega}{G r_{0}}\left(f_{1 v}{ }^{2}+f_{2 v}{ }^{2}\right)$

soit

$\mathrm{P}=-\frac{\mathrm{Gr}_{0}}{\omega} \frac{\mathrm{f}_{2 \mathrm{v}}}{\left(\mathrm{f}_{1 \mathrm{v}}{ }^{2}+\mathrm{f}_{2 \mathrm{v}}{ }^{2}\right)} \frac{\mathrm{dz}}{\mathrm{dt}}$

$+\mathrm{Gr}_{0} \frac{\mathrm{f}_{1 \mathrm{v}}}{\left(\mathrm{f}_{1 v}{ }^{2}+\mathrm{f}_{2 \mathrm{v}}{ }^{2}\right)} \mathrm{z}$

Cette équation peut être écrite plus simplement:

$\mathrm{P}=\mathrm{C}_{\mathrm{v}} \frac{\mathrm{d} z}{\mathrm{dt}}+\mathrm{k}_{\mathrm{v}} \mathrm{z}$

où

$C_{v}=-\frac{r_{0}^{2}}{a_{0}} \sqrt{\rho G} \frac{f_{2 v}}{f_{1 v}{ }^{2}+f_{2 v}{ }^{2}}$

et

$k_{v}=G r_{0} \frac{f_{1 v}}{f_{1 v}{ }^{2}+f_{2 v}{ }^{2}}$

$\mathrm{Si}$ la fondation a maintenant une masse $\mathrm{m}$ non négligeable et est soumise à une force périodique $Q$ ainsi qu'à la réaction - $\mathrm{P}$ du sol (fig. 3), son équilibre exige que:

$\mathrm{m} \frac{\mathrm{d}^{2} \mathrm{z}}{\mathrm{dt}^{2}}=\mathrm{Q}-\mathrm{P}$

soit

$\mathrm{m} \frac{\mathrm{d}^{2} \mathrm{z}}{\mathrm{dt}^{2}}+\mathrm{C}_{\mathrm{v}} \frac{\mathrm{dz}}{\mathrm{dt}}+\mathrm{k}_{\mathrm{vz}}=\mathrm{Q}=\mathrm{Q}_{0} \mathrm{e}^{\mathrm{i \omega t}}$

équation qui a la forme générale de l'équation d'un oscillateur simple.

On en déduit les caractéristiques générales du mouvement:

$A v=$ amplitude du mouvement vertical

$=\frac{\mathrm{Q}_{0}}{\mathrm{Gr}_{0}} \sqrt{\frac{\mathrm{f}_{1 \mathrm{v}}{ }^{2}+\mathrm{f}_{2 \mathrm{v}}{ }^{2}}{\left(1-b \mathrm{a}_{0}^{2} \mathrm{f}_{1 v}\right)^{2}+\left(b \mathrm{a}_{0}{ }^{2} \mathrm{f}_{2 v}\right)^{2}}}$

avec $\mathrm{b}=\mathrm{m} / \mathrm{\rho r}_{0}^{3}$

$\mathrm{A} \ddot{v}=$ amplitude des accélérations $=\omega^{2} \mathrm{Av}$

$\operatorname{tg} \varphi=$ déphasage entre la force $Q$ et le déplacement $z$

$=\frac{f_{2 v}}{-f_{1 v}+b a_{0}^{2}\left(f_{1 v}{ }^{2}+f_{2 v}{ }^{2}\right)}$

\section{DÉTERMINATION D'UN MODULE ÉQUIVALENT DU SOL RÉEL}

L'amplitude et le déphasage du mouvement de la fondation dépendent donc, pour une fréquence et une forme de fondation données, des constantes élastiques $\mathrm{G}$ et $v$ du sol supposé jusqu'ici homogène formules (7) et (8).

En ce qui concerne le coefficient de Poisson, et bien que ses variations affectent notablement les fonctions de déplacement (fig. 2), nous l'avons supposé constant et égal à une valeur de 0,25 qui nous a été imposée par les mesures des célérités des ondes dans le sol pour différentes accélérations statiques. 
Pour ce qui est du module de cisaillement, on peut supposer qu'il varie en fonction de la profondeur, ou plus exactement en fonction de la contrainte effective moyenne $\sigma_{\mathrm{m}}$, selon une loi proposée par Hardin et Richart [voir 3] à la suite d'essais dynamiques sur des sols pulvérulents soumis à une pression hydrostatique:

$$
G=g(e) \sqrt{\sigma_{m}^{\prime}}
$$

où $\mathrm{g}(\mathrm{e})$ est une fonction de l'indice des vides dont les coefficients varient avec la granulométrie des sols.

\subsection{Définition du module équivalent}

Nous allons définir un module $G$ équivalent pour notre sol rendu hétérogène par la pesanteur ainsi que par les contraintes dues à la fondation. Ayant mesuré la réponse Av̈ du modèle, la formule (7) - avec v = 0,25 - nous donne ce $G$ équivalent; nous montrerons qu'il peut être rapporté par une formule du type (9) à une contrainte caractéristique de la distribution de contrainte sous la fondation.

On remarque déjà, sur la figure 4 , que le $\mathrm{G}$ équivalent est constant pour une plaque et une accélération statique $\mathrm{g}$ donnée; la courbe de la figure 4 n'a pas été

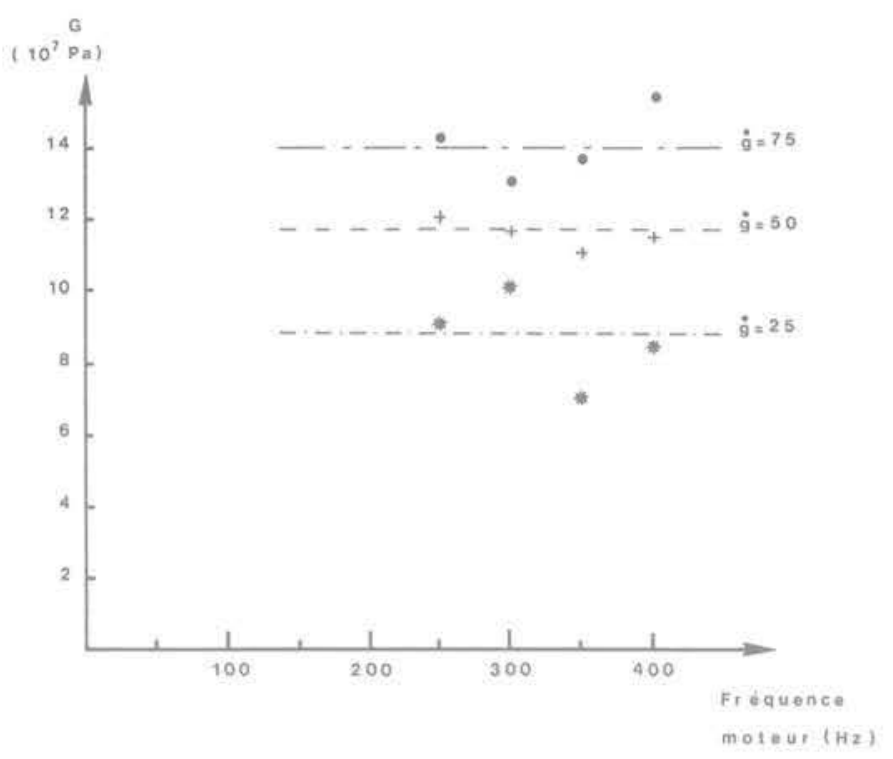

Fig. 4. - Valeur de $G$ équivalent pour la plaque $P_{1}$.

étendue vers les basses fréquences car les accélérations mesurées varient comme $\omega^{4}$ - formule (7') avec $Q_{0}$ variant comme $\omega^{2}$-; les autres résultats seront relatifs à une fréquence unique de $350 \mathrm{~Hz}$.

On remarque également que lorsque $\mathrm{g}^{*}$ double, le module $G$ équivalent est multiplié par $\sqrt{2}$ environ.

\subsection{La contrainte effective moyenne mini- male}

Pour ce qui est des applications aux ouvrages, Richart, Hall et Woods [3] conseillent de choisir comme $\sigma_{m}^{\prime}$ de la formule (9) la contrainte effective moyenne minimale, statique, sous la fondation et sur l'axe de celle-ci.

La contrainte effective moyenne sur l'axe est en effet la somme de la contrainte moyenne due au poids des terres (régime élastique avec $v=0,25$ ) et de la contrainte moyenne due au poids de la fondation que nous obtenons par les abaques classiques de Prange établis pour une semelle rigide.

Nous définissons ainsi une contrainte effective moyenne minimale $\sigma_{m}$ qui est, pour nos essais, proportionnelle à $\mathrm{g}^{*}$.

\subsection{Résultats des essais}

La figure 5 montre que, pour une large garmme de $\sigma_{m}^{\prime}$, on peut écrire $\mathrm{G}$ équivalent

$\mathrm{G}_{\text {equivalent }}^{*}=810^{5} \sqrt{\sigma_{m}^{\prime}}\left(\mathrm{G}\right.$ et $\sigma_{m}^{\prime}$ en $\left.\mathrm{Pa}\right)$

On peut remarquer que la valeur du coefficient de proportionnalité $\left(810^{5}\right)$ est très proche de celle proposée par Hardin et Richart pour un sable de même indice des vides et de même granulométrie.

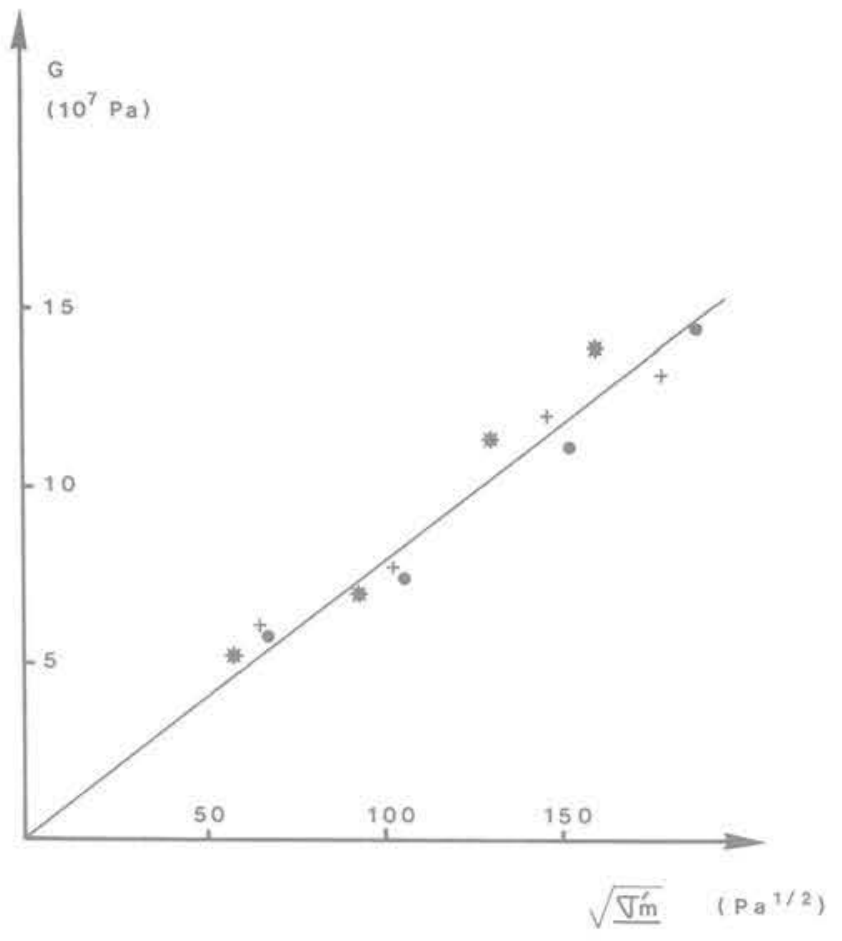

Fig. 5. - Variation de $G$ équivalent avec $\sigma_{\mathrm{m}}$.

\section{CONCLUSION}

Les résultats que nous venons d'exposer montrent donc qu'on peut caractériser l'ouvrage (fondation et sol) par une contrainte effective moyenne minimale, en déduire le module équivalent qui permet enfin de connaître la réponse de la fondation à une sollicitation dynamique. 


\section{BIBLIOGRAPHIE}

1. MANDEL, J. - Essais sur modèles réduits en mécanique des terrains. Etude des conditions de similitude. - Rev. Ind. Min., vol. 44, $\mathrm{n}^{\circ}$ 9. 1962.

2. DELEUZE, G. - Réponse à un mouvement sismique d'un édifice posé sur un sol élastique. Annales I.T.B.T.P., $n^{\circ}$ 234. 1967.

3. RICHART, F.E ; HALL, J.R.; WOODS, R.D. Vibrations of Soils and Foundations. - Prenctice Hall. 1970.
4. PILOT, G. - Centrifugation de modèles réduits d'ouvrages en terre et de fondations. - Rapport de Recherche, $n^{\circ} 48$, L.C.P.C. 1975.

5. ZELIKCSON, A.; LEGUAY, P.; PASCAL, C. Centrifugal model comparison of pile and raft foundations subjected to earthquakes. Soil Dynamics and Earthquake Engineering Conference. Southampton. 1982.

6. RIVIERE, J. - Interaction sol-structure lors d'un séisme; simulation en centrifugeuse. - Thèse $3^{e}$ cycle $n^{\circ} 1919$, Université Bordeaux 1. 1983. 